\title{
fissuration longitudinale des chaussées
}

par

Alfonso Rico Rodriguez

Dans plusieurs pays d'Amérique latine, d'Afrique et d'Asie, il est fréquent d'observer une rupture caractéristique des nouvelles routes ou des routes reconstruites, élargies ou aménagées pour les exigences croissantes du trafic routier. Cette rupture est mise en évidence par la formation de fissures, très rapprochées les unes des autres, qui se développent systématiquement à 1 ou 2 mètres des bords du remblai près de la surface de roulement. Ces fissures s'étendent longitudinalement sur des centaines de mètres, voire sur des kilomètres, suivant une direction régulière généralement des deux côtés de la route.

Si l'on ne prend pas des mesures préventives, la largeur et la profondeur de ces fissures augmentent et d'autres fissures peuvent apparaître parallèlement aux premières. L'effet de l'eau de pluie qui remplit ces fissures est probablement la cause principale de leur développement qui a pour résultat la rupture des talus par la pression hydrostatique de l'eau. Un effet secondaire d'importance est la destruction de l'uniformité de la surface du remblai par désintégration des lèvres des fissures. II a été observé également que ces fissures ont une tendance à se propager vers le centre de la chaussée, détruisant ainsi la surface de roulement. En résumé, les fissures longitudinales peuvent détruire une route si des mesures correctives ne sont pas prises à temps.

II a déjà été dit que cette détérioration peut être observée aussi bien sur les nouvelles routes que sur celles qui ont été reconstruites, renforcées ou élargies. Dans ce dernier cas, la fissuration est due à l'influence du compactage.

En ce qui concerne l'Amérique latine, et dans la limite de l'expérience personnelle de l'auteur, le compactage a été très peu ou pas du tout employé dans la construction routière. Au Mexique, par exemple, la première route compactée a été réalisée en 1950. De ce fait, une grande proportion des anciennes routes, modernisées ou élargies de nos jours, ont été compactées d'une façon très insuffisante. II est bien connu que le compactage augmente la tendance des sols fins (ou des sols ayant une grande proportion de particules fines) à la fissuration. En conséquence on peut observer actuellement des fissures sur les chemins aménagés ou modernisés et qui ont été mis en service depuis plus de 25 ans sans avoir jamais montré de fissuration longitudinale apparente. II est évident que l'élargissement et le renforcement sont faits avec des techniques modernes de construction qu'on considère comme adéquates. De tels accidents engendrent évidemment des inconvénients structurels, mais, et c'est peut-être plus important, ils remettent en question les nouvelles technologies et les techniciens, car des chemins modestes avec des niveaux de service acceptables sont ruinés lorsqu'on essaie de les réparer ou de les remplacer par d'autres de meilleure apparence ou plus larges et qui sont détruits rapidement.

Pour mieux comprendre les causes fondamentales du problème de fissuration longitudinale, il est nécessaire de faire un aperçu de l'évolution des politiques de compactage des sols en Amérique latine et faire quelques remarques sur les matériaux utilisés pour la construction routière.

II y a à peine 30 ou 35 ans, les ingénieurs pensaient que le compactage des remblais n'était pas nécessaire. Quelques-uns d'entre eux considéraient le compactage comme une technique à la mode dont le coût ne correspondait en aucun cas au profit qu'on en tirait. En fait cette technique a été considérée comme exotique et soumise à l'opposition naturelle aux nouveautés. II est clair que cette attitude ne pouvait pas durer longtemps et le compactage a été rapidement accepté à tous les stades en devenant une méthode essentielle pour la construction des routes. La définition initiale du compactage comme une méthode d'amélioration de la densité des sols, ajoutée à la méconnaissance des diverses méthodes de compactage, ce qui est très courant spécialement pour les personnes qui travaillent sur chantier, ont fait évoluer les façons de penser 
et on a atteint ce qu'on peut appeler "l'âge du compactage à tout prix $m$. On avait l'idée que plus un matériau est compacté, meilleures sont les propriétés que l'on peut en tirer, indépendamment de toute autre considération. Le compactage n'était pas utilisé comme une des méthodes pour améliorer le comportement mécanique d'un sol, mais très souvent il était, malheureusement, la seule qui soit prise en compte.

On doit citer une autre condition importante pour définir l'environnement de la construction routière en Amérique latine. Les réseaux nationaux supportaient traditionnellement des niveaux de trafic très bas; de nos jours la situation a changé et au Mexique, par exemple, il y a des autoroutes représentant moins de $25 \%$ du réseau total, avec un trafic journalier de plus de 20000,50000 et même 150000 véhicules. Dans d'autres pays d'Amérique latine le trafic est encore plus petit et il est fréquent de faire les projets de routes pour un trafic estimé à 500 à 1000 véhicules par jour.

D'autre part, on est très conscient du rôle social et politique joué par les transports dans les pays en voie de développement. Cette prise de conscience oblige à rechercher essentiellement la minimisation du prix initial des travaux, car on sait bien qu'il y a beaucoup à faire pour soulager toutes les déficiences ancestrales de l'état social. On peut penser que ce critère vise trop loin avec des conséquences sévères car, malgré le grand soin apporté au coût initial des autoroutes, on néglige d'autres aspects importants tels que l'entretien et l'exploitation. Dans des pays comme le Mexique, avec un développement extrêmement croissant, ce critère a conduit à un réseau routier non souhaitable dans les conditions actuelles et qui sera une source de problèmes dans l'avenir où l'on prévoit des trafics et des charges importants. En conclusion, on voit que divers aspects n'ont pas été suffisamment considérés dans le projet et la construction des réseaux nationaux de transport, car on a considéré que le point le plus important était de minimiser le prix initial de chaque route.

Le résultat de cette façon de penser est l'emploi de matériaux de mauvaise qualité, qui ne conviennent pas pour la construction des routes. L'utilisation de sols très plastiques avec des particules fines est courante et, d'autre part, la pratique du drainage visant à protéger les massifs de sols des terrassements, ainsi que le revêtement, n'est pas très courante. Finalement, l'emploi de sols stabilisés dans les routes est très rare.

Considérons les conséquences de la coexistence de ces deux critères.

L'emploi fréquent de matériaux de qualité douteuse et l'idée que le compactage est meilleur lorsque les niveaux d'énergie appliquée sont plus grands conduisent à des problèmes d'instabilité volumique sous l'effet de l'eau dans les terrassements, et naturellement à des problèmes de fatigue qui concernent $25 \%$ du réseau routier.

C'est dans ce panorama que le problème de la fissuration longitudinale des remblais en Amérique latine doit être examiné.

Le mécanisme de fissuration a été conçu comme suit. a) En chaque point du sol, préalablement à la construction d'une route, il existe un équilibre hydraulique entre l'évaporation superficielle et l'écoulement d'eau des couches inférieures, soit par capillarité, soit par remontée de la nappe phréatique pendant la saison des pluies. La précipitation pluviale dans la région est un élément qui participe ainsi à cet équilibre global. b) Le régime hydraulique est modifié lorsque le remblai est construit, empêchant l'évaporation du sol juste sous l'ouvrage. De ce fait la teneur en eau du sol dans les zones imperméables tend à augmenter si le climat comporte une action solaire intense.

c) Si le remblai possède une grande proportion de particules fines, la teneur en eau augmentera par infiltration de l'eau en provenance des couches inférieures, spécialement pendant les saisons de pluies. D'autre part, et surtout pendant la saison sèche, l'eau du remblai s'évapore sous l'effet de l'intense action solaire. Ces phénomènes ne sont cependant pas uniformes dans tout le corps du remblai; les talus sont les zones les plus sensibles puisqu'ils sont les plus exposés à l'air, contrairement aux parties centrales plus protégées, surtout lorsqu'il y a un tapis, d'où un effet différentiel d'évaporation entre les matériaux qui se trouvent sur les côtés et ceux du centre du remblai. II est courant d'observer pendant la période sèche que la teneur en eau des talus est réduite considérablement, tandis que dans la partie centrale elle reste pratiquement constante à toute époque; dans certains cas, on a même pu rapporter des augmentations de la teneur en eau des zones centrales en période sèche.

d) Lorsqu'un sol ayant beaucoup de particules fines perd son eau par évaporation, il en résulte un processus de contraction volumique; l'évaporation différentielle est donc accompagnée par son équivalent de retrait différentiel. Ce retrait est plus important dans les talus que dans la partie centrale du remblai, ce qui produit des fissures d'autant plus grandes que les sols utilisés sont plus sensibles aux variations volumiques par séchage. Si l'on considère une section droite quelconque du remblai, la zone fissurée apparaîtra quelque part entre le centre et les épaulements de la route; puisque toutes les sections droites voisines sont dans des conditions similaires, les zones de fissuration seront pratiquement en coïncidence. Dans la chaussée, considérée globalement, deux zones fissurées longitudinalement apparaîtront de chaque côté du remblai et dans la même direction.

e) Lorsque le dernier mécanisme est raisonnable, les fissures apparaissent au début de la saison sèche d'une façon relativement brutale.

f) II est bien connu que jusqu'à un certain niveau au-dessus de la nappe phréatique, le sol contient de l'eau qui remplit pratiquement complètement les vides et à partir de ce niveau le degré de saturation décroît lorsqu'on s'éloigne de la nappe. Toute cette eau interstitielle est dans un état de traction décroissante avec le degré de saturation. En outre, dans la zone partiellement saturée, il y a de la vapeur d'eau dont la pression peut être réduite par l'évaporation superficielle ou par une chute de la température.

Dans la partie basse de cette zone, près de la limite où le phénomène capillaire engendre une saturation presque complète, il y a toujours continuité dans l'eau mais, puisque cette eau ne remplit pas tous les vides, les contraintes effectives ne seront plus égales à la différence entre les contraintes totales et la pression interstitielle. Dans la partie haute de la zone partiellement saturée, l'eau ne présente pas de continuité et le degré de saturation décroit rapidement vers la surface du sol. Les mouvements de la vapeur d'eau dépendront du gradient de pression de vapeur qui peut exister. Si la pression de vapeur augmente, par exemple à cause d'une montée de la température ambiante, l'eau a tendance à remonter, ce qui réduit la couche de sol partiellement saturé. Cependant, en fonction de l'environnement extérieur et du type de sol. du fait que 
dans la frontière supérieure en contact avec l'air la tension capillaire et les conditions sont les mêmes que dans les zones voisines, la distribution de la pression dans l'eau est plus grande que celle qui correspondait à une loi linéaire et est seulement équilibrée par uné réduction du rayon des unénisques d'eau entre les grains. Un écoulement continu vers la surface s'établit ainsi, comme par exemple dans les zones désertiques pendant les périodes sèches sous une action solaire intense.

Si l'on considère les conditions et le mécanisme de rupture cités ci-dessus, il apparaît que la fissuration longitudinale se produit lorsque l'on a :

a) une alternance des saisons sèches et pluvieuses, avec une forte action solaire pendant les périodes sèches;

b) une action capillaire intense, dans le sol naturel d'assise et dans le remblai lui-même. La nappe doit être proche de la surface du sol naturel comme c'est le cas des zones plates ou des dépressions;

c) une utilisation de sols susceptibles d'avoir d'importants changements volumiques lorsque la teneur en eau varie.

Ces conditions sont très fréquentes au Mexique. Si en plus elles sont accompagnées de l'utilisation dans les ouvrages en terre de sols ayant une proportion considérable de particules fines et par l'emploi systématique du compactage, on comprend pourquoi la fissuration longitudinale est un sérieux problème pour les routes mexicaines. Ceci explique en même temps l'intérêt des ingénieurs mexicains pour ce sujet, qui a été étudié depuis plus de dix ans par «El Secretaria de Asentamientos Humanos y Obras Públicas" et par "I'Instituto de Ingeniería" de I'Université Nationale.

Trois objectifs ont guidé les travaux de recherche de ces deux organismes:

a) connaître les conditions de chantier qui mènent à la fissuration, savoir comment la fissuration évolue et proposer des mesures pour la corriger lorsqu'elle est déjà visible, ou pour la prévenir. ou pour l'éliminer dans les nouveaux projets;

b) déterminer expérimentalement quels sont les sols les plus couramment associés au problème de fissuration et établir des critères pour les dépister en développant des méthodes de classification des sols en fonction de leur sensibilité à ce phénomène;

c) confirmer expérimentalement les mécanismes qui engendrent ce problème et déterminer leurs corrélations quantitatives avec les conditions sur chantier et leur évolution.

\section{Recherche sur chantier}

Compte tenu des brusques changements de climat entre les saisons sèches et les saisons fortement pluvieuses, couramment observés au Mexique, on s'est intéressé à la réponse des sols et des remblais aux changements volumiques. Considérons les dilatations d'une route typique construite sur un sol argileux, présentées sur la figure 1; il s'agit d'un remblai de $1,5 \mathrm{~m}$ de hauteur constitué par un sol ayant une grande proportion de particules fines. Le sol naturel a subi des gonflements assez notables à la fin de la période des pluies (ligne en pointillé) par rapport à la position initiale rencontrée à la fin de la période sèche (ligne en trait continu). On remarquera que la tranchée latérale est trop près de la structure et qu'elle a été inondée. On remarquera aussi que le corps du remblai n'a pas bougé par rapport aux talus et au sol naturel d'assise. Ce schéma est en bon accord avec les hypothèses indiquées précédemment.

Plusieurs études in situ ont porté sur les variations de la teneur en eau des remblais et des sols d'assise, avec des carottages et des essais de laboratoire.

La figure $2 \mathrm{a}$ correspond à un remblai de $4 \mathrm{~m}$ de haut construit avec un sable argileux, issu de la décomposition et de la désintégration partielle d'un schiste. La partie centrale du remblai est constituée par une argile, produit de décomposition totale de la même roche; le sol d'assise est composé de cette même argile. Ceci est un cas de modernisation et d'élargissement et le remblai argileux initial est couvert et protégé par un sable argileux. Les chiffres entre parenthèses indiquent les teneurs en eau à la fin de la construction. correspondant dans ce cas à la fin de la saison des pluies.

A cette première étape, la surface du remblai n'a pas été protégée. Le sable argileux a été compacté à $95 \%$ de la densité sèche maximale Proctor Standard, avec $17.5 \%$ de teneur en eau optimale et l'argile inférieure a été compactée à $90 \%$ du Proctor Standard et avec une teneur en eau optimale de $37,5 \%$.

La figure $2 \mathrm{~b}$ montre le mềme remblai non protégé à la fin de la période sèche. Les chiffres entre parenthèses représentent toujours les teneurs en eau mesurées et les chiffres sans parenthèses correspondent aux variations volumiques en pourcentage observées dans le remblai. On notera que le remblai argileux initial n'a pas eu de changements sensibles, comparés à ceux du sol d'assise et du sable argileux.

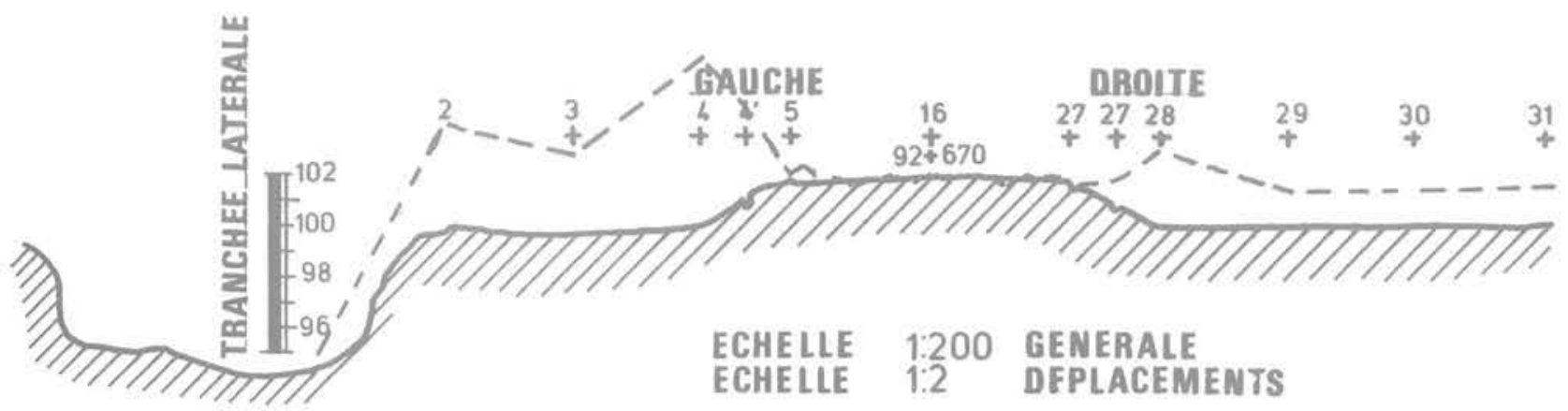

Fig. 1 Déplacements du sol dans une section naturelle 


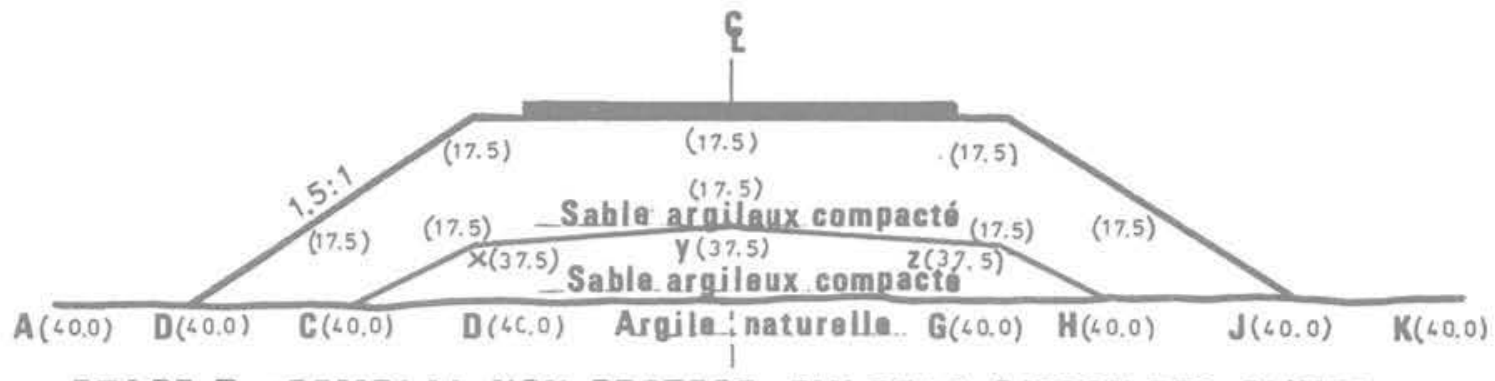

\section{ETAPE I :REMBLAI NON PROTEGE_FIN DE LA_SAISON DES PLUIES}

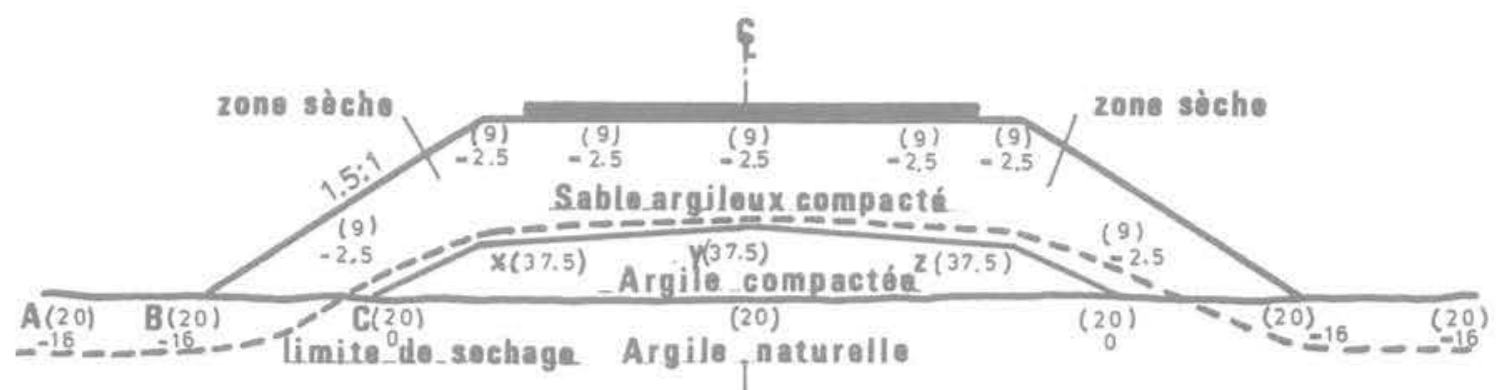

ETAPE II : REMBLAI NON PROTEGE _ FIN DE LA SAISON SECHE
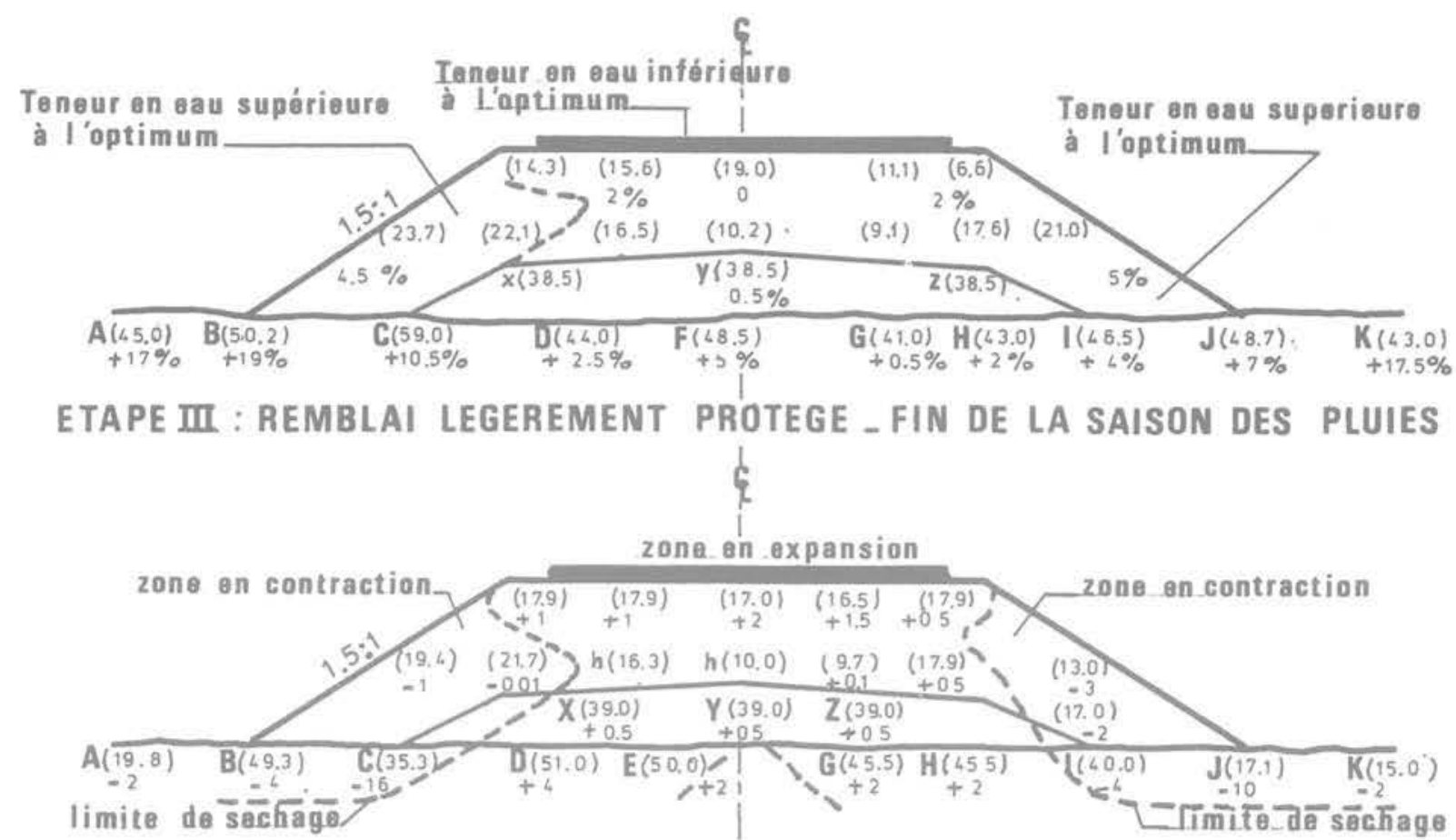

ETAPE I : REMBLAI AVEC UN RECOUVREMENT d'ASPHALTE_FIN DE LA SAISON SECHE

$\begin{array}{lllllll}0 & 1 & 2 & 3 & 6 & 5 & \mathrm{~m}\end{array}$

\section{ECHELLE \\ Fig. 2 Séquence des changements de volume d'un remblai routier}

A la fin de cette saison sèche la surface de roulement a été protégée et les conditions montrées sur la figure $2 \mathrm{c}$ correspondent à la fin de la saison des pluies suivante, avec la même interprétation pour les chiffres entre parenthèses. Les chiffres sans parenthèses indiquent les variations volumiques qui sont maintenant des dilatations, dont les plus importantes ont eu lieu dans les talus et dans le sol d'assise, surtout aux endroits où il n'y a pas de protection. On observe également que la teneur en eau à l'intérieur du remblai reste inférieure à la teneur optimale tandis que dans le talus elle est considérablement plus grande. Le potentiel capillaire peut expliquer la faible augmentation de la teneur en eau initiale du remblai argileux par rapport à la teneur en eau naturelle d'origine. Immédiatement après, le remblai a été recouvert par une couche d'asphalte et la figure $2 \mathrm{~d}$ montre les mesures effectuées à la fin de la saison sèche suivante. On a conservé la présentation des résultats par des chiffres entre parenthèses, mais les chiffres sans parenthèses correspondent à un retrait lorsque le signe est négatif et à un gonflement dans le cas contraire. II est évident qu'à la fin de la saison sèche la teneur en eau de ce remblai protégé par une couche d'asphalte est restée la même ou a 


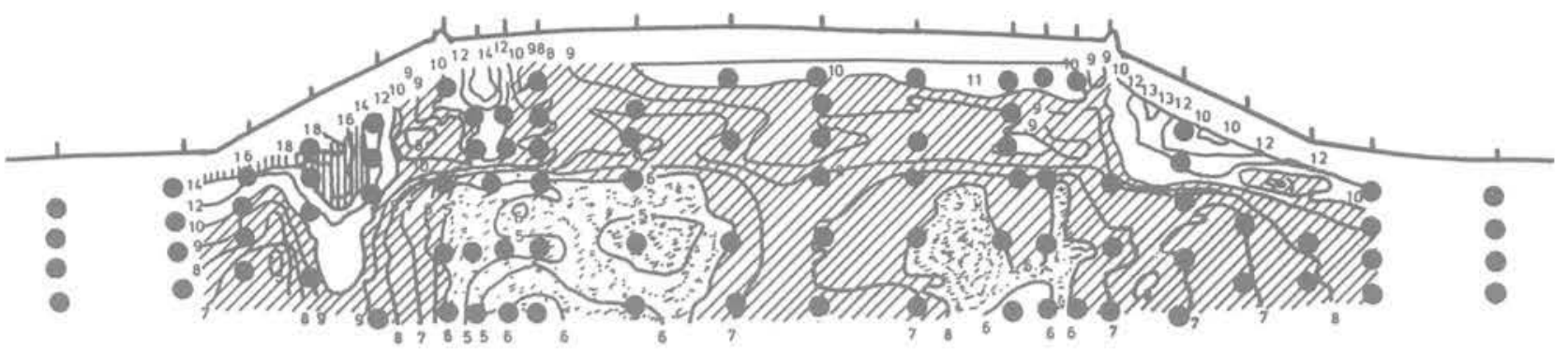

- POINT DE MESURE

FIN DE LA SAISON SECHE

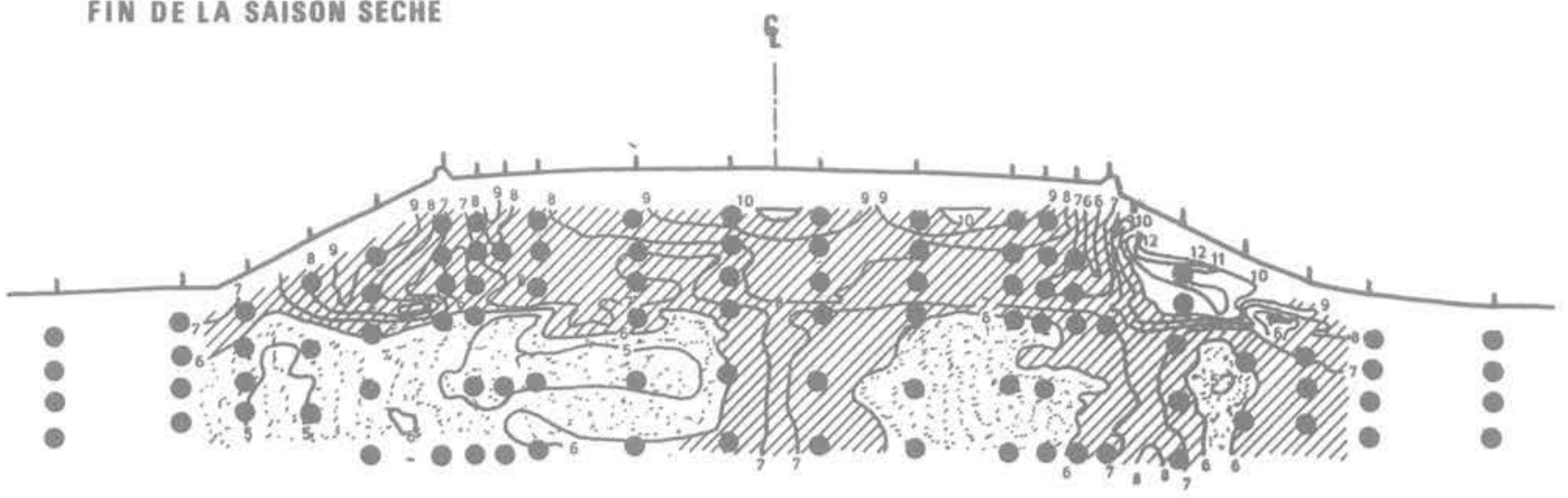

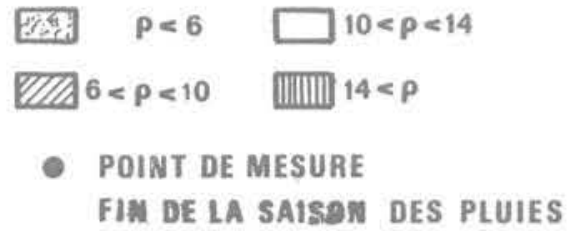

Fig. 3 Mesures caractéristiques de la résistivité dans un remblai routier

légèrement augmenté, alors que les zones de talus se sont desséchées, d'où la présence d'un processus de retrait. Dans le sol d'assise la zone non protégée par le remblai s'est considérablement desséchée.

Ces résultats représentatifs de toutes les sections étudiées viennent corroborer les hypothèses de base formulées pour expliquer le phénomène.

Une autre étude in situ qui doit être mentionnée est l'ensemble de mesures de résistivité électrique réalisées dans plusieurs sections de contrôle pratiquées sur différentes routes fissurées. Le but était de déterminer indirectement la teneur en eau en plusieurs endroits et ses variations saisonnières avec une méthode non destructive. La méthode de mesure est classique avec l'installation d'électrodes dans le corps du remblai. Sur la figure 3 on présente un remblai de $14 \mathrm{~m}$ de long et $3 \mathrm{~m}$ de haut, construit avec un matériau dont la teneur en argile est de $35 \%$. Le sol d'assise est argileux.

La figure $3 a$ montre les résultats des mesures de résistivité à la fin de la période sèche et la figure $3 \mathrm{~b}$ correspond à la fin de la saison des pluies. On observe à nouveau que le dessèchement a lieu dans les talus et qu'il est négligeable au centre du remblai. Le sol d'assise tend également à se dessécher par évaporation dans les parties qui ne sont pas protégées par le remblai. Dans les zones des talus, la résistivité peut augmenter dans la proportion du simple au double à la fin de la saison sèche, ce qui révèle une réduction correspondante de la teneur en eau.

\section{Recherche en laboratoire}

Un programme de recherches très étendues a été effectué depuis quinze ans; la série de travaux réalisés au Secretaria de Obras Públicas (sous la direction de l'auteur et de M. R. V. Orozco) et à I'Instituto de Ingeniería (M. J. Alberro) sera présentée maintenant.

\subsection{Travaux de MM. Rico et Orozco}

On considère deux matériaux utilisés couramment pour la construction de routes dans la zone du Yucatán : le premier est une argile fortement compressible et l'autre est un limon ou stable argileux calcaire, tous deux résultats de la décomposition et de la désintégration d'une roche calcaire caractéristique de cette zone karstique du Yucatán. Le matériau argileux est le plus difficile à traiter mais il est parfois utilisé pour la reconstruction des remblais. Le sable est le matériau de base pour la construction initiale des remblais. Le tableau de la figure 4 résume quelques propriétés des deux matériaux.

Les essais de gonflement réalisés avec le dilatomètre Hveem ont permis de déterminer ce qu'on appelle les " trajets compactage en fonction de la teneur en eau ". Ces courbes sont construites comme suit : à partir de la densité sèche maximale Proctor pris comme état de référence, on calcule les pourcentages de compactage en faisant le rapport entre le poids volumique à un instant donné et la valeur de référence. 


\begin{tabular}{|c|c|c|c|c|c|c|c|c|c|c|c|c|}
\hline Sol & Echantillon & $\begin{array}{l}\mathrm{LL} \\
\%\end{array}$ & $\begin{array}{l}\text { LP } \\
\%\end{array}$ & $\begin{array}{l}I_{p} \\
\%\end{array}$ & $\begin{array}{l}\mathrm{LR} \\
\%\end{array}$ & $\begin{array}{c}\text { Colloides } \\
\%\end{array}$ & $\begin{array}{l}s_{5} \\
\%\end{array}$ & $\begin{array}{l}\text { M.0. } \\
\%\end{array}$ & $\begin{array}{c}\mathrm{CO}_{3} \mathrm{Na}_{2} \\
\%\end{array}$ & $\begin{array}{c}\mathrm{CO}_{3} \mathrm{HNa} \\
\%\end{array}$ & $\begin{array}{c}\text { CLNa } \\
\%\end{array}$ & $\begin{array}{l}\text { Potentiel de } \\
\text { gonflement d'après } \\
\text { Holtz et Gibbs }\end{array}$ \\
\hline $\begin{array}{l}\text { Sable } \\
\text { argileux }\end{array}$ & remanié & 31 & 20 & 11 & 18 & 7 & 2,74 & 2 & 70 & 0,7 & 0,06 & modéré à moyen \\
\hline $\begin{array}{l}\text { Argile } \\
\text { naturelle }\end{array}$ & intact & $\begin{array}{r}94 \\
111 \\
112\end{array}$ & $\begin{array}{l}37 \\
38 \\
34\end{array}$ & $\begin{array}{l}57 \\
73 \\
78\end{array}$ & $\begin{array}{r}13 \\
3 \\
10\end{array}$ & $\begin{array}{l}59 \\
74 \\
37\end{array}$ & $\begin{array}{l}2,39 \\
2,36 \\
2,38\end{array}$ & $\begin{array}{l}22 \\
19 \\
12\end{array}$ & $\begin{array}{l}- \\
-\end{array}$ & $\begin{array}{l}0,7 \\
0,4 \\
0,7\end{array}$ & $\begin{array}{l}0,07 \\
0,08 \\
0,06\end{array}$ & $\begin{array}{l}\text { élevé à très élevé } \\
\text { très élevé } \\
\text { très élevé }\end{array}$ \\
\hline $\begin{array}{l}\text { Argile } \\
\text { compactée }\end{array}$ & remanié & $\begin{array}{r}88 \\
103 \\
125 \\
95 \\
98 \\
122 \\
115 \\
116\end{array}$ & $\begin{array}{l}36 \\
37 \\
41 \\
34 \\
29 \\
26 \\
28 \\
31\end{array}$ & $\begin{array}{l}52 \\
56 \\
84 \\
61 \\
69 \\
96 \\
87 \\
85\end{array}$ & $\begin{array}{l}- \\
\overline{-} \\
\overline{-} \\
\overline{-} \\
\overline{-}\end{array}$ & $\begin{array}{l}56 \\
66 \\
64 \\
- \\
- \\
- \\
- \\
-\end{array}$ & \begin{tabular}{c|}
- \\
- \\
- \\
2,48 \\
2,45 \\
2,39 \\
2,38 \\
2,40
\end{tabular} & $\begin{array}{l}- \\
- \\
- \\
- \\
- \\
-\end{array}$ & $\begin{array}{l}- \\
- \\
- \\
- \\
- \\
- \\
-\end{array}$ & $\begin{array}{l}- \\
- \\
- \\
- \\
- \\
-\end{array}$ & $\begin{array}{l}- \\
- \\
- \\
- \\
- \\
-\end{array}$ & $\begin{array}{l}\text { élevé à très élevé } \\
\text { très élevé } \\
\text { très élevé } \\
\text { élevé à très élevé } \\
\text { élevé à très élevé } \\
\text { très élevé } \\
\text { très élevé } \\
\text { très élevé }\end{array}$ \\
\hline
\end{tabular}

Fig. 4 Propriétés des sols étudiés

LL limite de liquidité

LP limite de plasticité

$I^{p}$ indice de plasticité

$L R$ limite de retrait

$S_{S}$ densité

MO teneur en matière organique

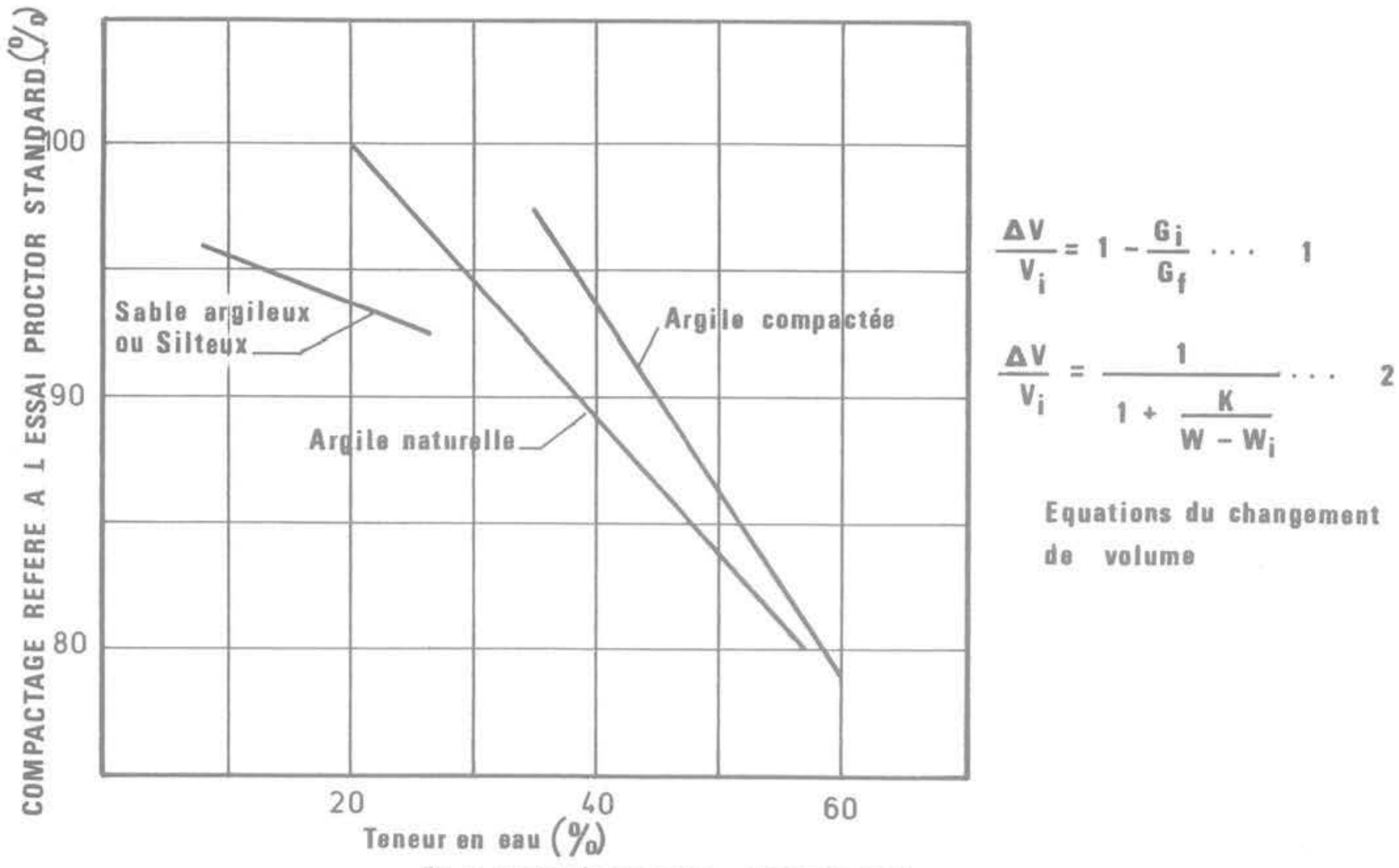

Fig. 5 Trajets (compactage - teneur en eau)

Différents échantillons de sable et d'argile ont été compactés du " côté sec " et placés dans le dilatomètre Hveem, en les laissant se dilater librement jusqu'à ce que la condition finale appelée saturation soit atteinte. Dans tous les cas l'évolution volumique a été observée en fonction des variations de la teneur en eau et cette évolution, qui est obtenue pour chaque échantillon essayé, est appelée "trajet .

Un exemple représentatif de trajet pour ces sols est montré sur la figure 5. II est clair que plus la courbe est aplatie, moins le sol est sensible aux variations volumiques. II faut observer que la plupart des courbes expérimentales sont assez proches de celles qui sont présentées ici comme des courbes typiques. Pour le sable et l'argile compacte, les trajets obtenus sont des droites et ceux de l'argile naturelle sont légèrement courbés, dans les trajets représentatifs de la figure 5, on a volontairement omis cette courbure.

La formule 1 ci-dessous exprime les variations volumiques du sol en fonction des degrés de compactage (initial et final) de chaque échantillon de sol. Le degré de compactage final est exprimé par rapport à la densité sèche maximale Proctor:

$$
\frac{\Delta V}{V_{i}}=1-\frac{G_{i}}{G_{i}}
$$


L'application de cette formule à un cas réel est faite à partir d'un trajet faisant intervenir les conditions de compactage initial et la teneur en eau du sol. Dans un tel cas les trajets fournissent un critère pour évaluer le pourcentage de variation volumique du sol en fonction de la teneur en eau. Ces trajets ont ainsi été utilisés avec succès à divers projets pour estimer le comportement futur de l'ouvrage.

A partir de l'expression (1) on obtient facilement la formule (2) où w est la teneur en eau maximale prévue pour le remblai étudié, et $w_{1}$ la teneur en eau initiale du même matériau; $w_{i}$ sera en général la teneur en eau optimale de compactage in situ; $\mathrm{K}$ est un paramètre adimensionnel qui dépend de la pente du trajet envisagé et du degré de compactage initial du sol.

$$
\frac{\Delta V}{V_{1}}=\frac{1}{1+\frac{K}{w-w_{1}}}
$$

L'intérêt de cette expression est qu'elle permet de donner un critère de projet pour déterminer le degré de compactage et la teneur en eau pour qu'un sol devant être placé dans un remblai minimise sa sensibilité aux changements volumiques. Pour illustrer cela, la figure 6 montre les courbes de $\mathrm{K}$ en fonction de la teneur en eau initiale pour le sable argileux ou silteux cité précédemment. Les courbes ont été obtenues au laboratoire avec un dilatomètre qui mesure les variations volumiques du sol en fonction des variations de la teneur en eau à partir d'une valeur initiale donnée, jusqu'à la valeur finale, c'est-à-dire de saturation dans le dilatomètre.

Si la teneur en eau optimale de compactage du matériau sur le chantier est par exemple de $17,5 \%$, la figure montre qu'à $96 \%$ du compactage initial la valeur de $\mathrm{K}$ est de 6,5 , mais que pour $100 \%$ elle est réduite à 3,5 . Avec ces deux valeurs de $K$ et avec une estimation de la teneur en eau que le sol peut atteindre dans le remblai, la formule 2 permet de calculer les variations volumiques correspondant à ces deux degrés de compactage: dans ce cas particulier le compactage à $96 \%$ est meilleur que celui à $100 \%$.

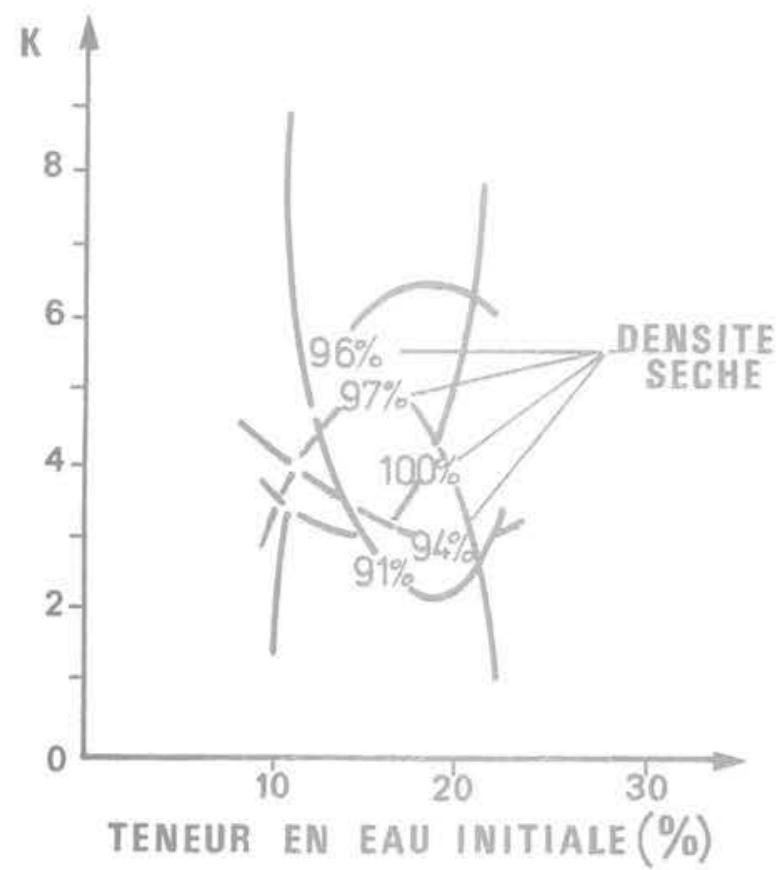

Fig. 6 Valeurs de $K$ pour des teneurs en eau et des degrés de compactage différents
Vu la forme compliquée des courbes donnant la valeur de K, on peut dire qu'il n'y a pas de relation apparente sous forme d'expression simple entre le degré du compactage donné au sol du remblai et les variations volumiques pour chaque sol et pour chaque condition particulière. Ce fait, qui confirme l'expérience des praticiens, indique qu'il est nécessaire de faire pour chaque projet l'étude détaillée qui vient d'être décrite ici.

\section{$2: 1$ Travail de M. Alberro}

Le but de ce travail était de déterminer la sensibilité aux déformations volumiques des sols utilisés couramment pour la construction des remblais en fonction de leur teneur en eau initiale et de leur poids volumique.

Deux matériaux ont été considérés, ils sont dénommés $\mathrm{CH}$ dans la classification de Casagrande, avec $\mathrm{LL}=85 \%$ et $79 \%$ respectivement. $\mathrm{IP}=55 \%$ et $47 \%$ et LR de $13,7 \%$ dans les deux cas.

La recherche a été réalisée en laboratoire sur des éprouvettes compactées avec une énergie spécifique de $7,5 \mathrm{~kg} \cdot \mathrm{cm} / \mathrm{cm}^{3}$ pour le premier matériau et de 1 ; 3,$3 ; 7,5$ et $30,1 \mathrm{~kg} \cdot \mathrm{cm} / \mathrm{cm}^{3}$ pour le deuxième. Des essais de dilatation volumique ont été effectués en même temps que des essais triaxiaux sur ces éprouvettes en faisant diverses combinaisons des paramètres suivants : énergie de compactage, teneur en eau initiale, pression de confinement et aussi contrepression interstitielle.

La figure 7 présente les dilatations volumiques du deuxième matériau compacté à trois niveaux différents d'énergie en fonction de la teneur en eau initiale, et la figure 8 présente la dilatation volumique des mêmes échantillons en fonction de la pression effective de confinement employée dans l'essai triaxial.

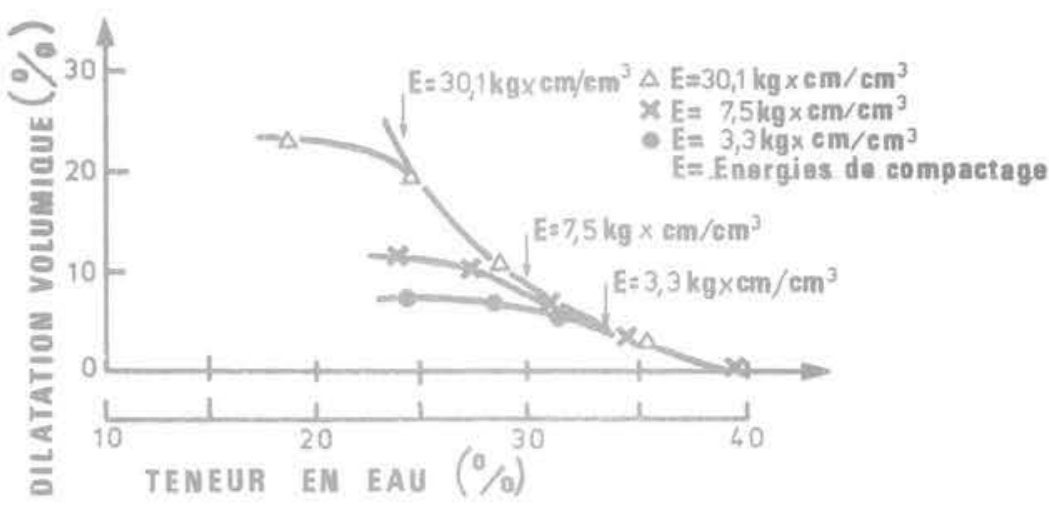

Fig. 7 Gonflement en fonction de la teneur en eau initiale

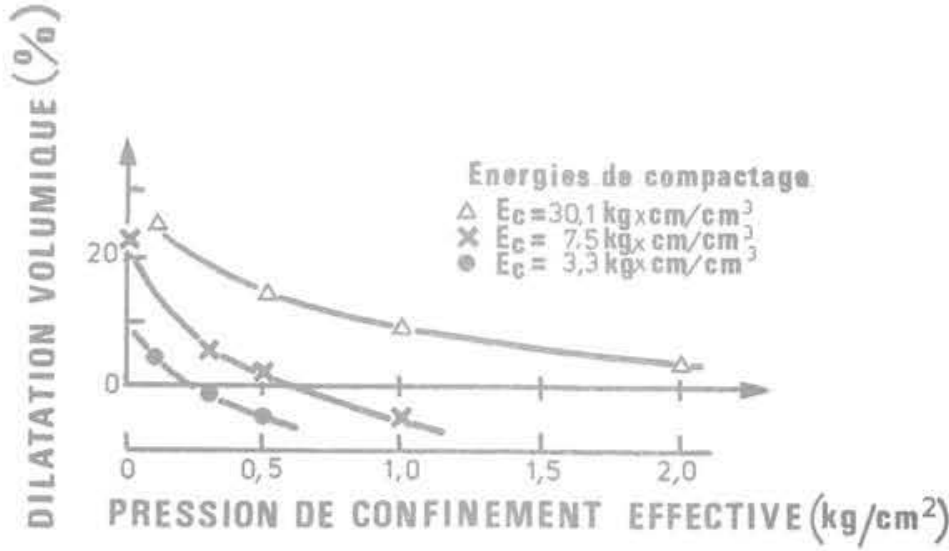

Fig. 8 Gonflement en fonction de la pression effective 
Les essais de dilatation ont été complétés par des essais de retrait par un séchage lent.

Les résultats importants de cette recherche sont les suivants :

\section{a) Influence du poids volumique initial}

Pour une même teneur en eau de compactage, les échantillons les plus denses ont montré un plus grand gonflement, ce qui est corroboré par d'autres résultats rapportés dans la littérature (fig. 7). Les courbes de dilatance ont une enveloppe commune, ce qui implique que le gonflement de tous les échantillons avec une teneur en eau supérieure à l'optimum dépend essentiellement de la teneur en eau de compactage.

b) Influence de la teneur en eau de compactage

La dilatation volumique augmente lorsque la teneur en eau de l'échantillon décroît. Ce résultat a déjà été obtenu par d'autres chercheurs.

c) Influence de la structure de l'échantillon

II est connu que les sols naturels prélevés de façon à rester intacts sont moins dilatants que les sols compactés à la même teneur en eau. II est connu. aussi, que la structure d'un sol compacté est liée à la position du sol sur la courbe de compactage. Ce fait a été traité largement dans la littérature, par exemple Maranha das Neves à Lisbonne ou Lambe au M. I. T. ont suggéré qu'il se forme une structure floculée dans les sols compactés avec une teneur en eau inférieure à l'optimum et que cette structure devient de plus en plus orientée lorsque la teneur en eau dépasse le point optimal. La recherche faite au Mexique pour étudier le comportement des sols fins compactés suggère que la description de la structure de ce type de sols en fonction de la position et des relations entre les particules, prises individuellement, n'est pas possible et que la structure des sols fins compactés est principalement une question d'assemblage et de liens entre des mottes, des groupes de particules et non des particules isolées. Actuellement des travaux sont effectués au Mexique pour déterminer les structures qui constituent les groupes floculés de particules. II semble que pour des teneurs en eau supérieures à l'optimum, tous les sols fins ont pratiquement la même structure, indépendamment de l'énergie de compactage; au contraire, pour les échantillons compactés à des teneurs en eau inférieures à l'optimum, la structure des floculats dépend fortement de l'énergie de compactage. On peut dire alors que les grandes énergies induisent de fortes pressions interstitielles par osmose, ce qui du même coup engendre de grands gonflements lorsque les pressions sont dissipées par humidification.

\section{d) Influence de la pression de confinement}

La dilatation d'un sol compacté à une teneur en eau et une densité données est réduite notablement lorsque la pression effective de confinement augmente (fig. 8), ce qui réduit l'équilibre de la teneur en eau. II en résulte que lorsque le sol est mis en contact avec de l'eau libre le degré de saturation de l'échantillon augmente sans changement de volume.

\section{e) Influence du temps de repos}

Lorsque le temps entre la préparation de l'échantillon à la teneur en eau de compactage et le compactage proprement dit augmente, le potentiel de gonflement décroît. Ce phénomène doit être relié aux propriétés de thixotropie des argiles. Puisque la résistance au cisaillement des sols augmente avec le compactage, l'efficacité de l'énergie décroît et l'on obtient des poids volumiques moins grands; le potentiel de gonflement correspondant se trouve donc diminué.

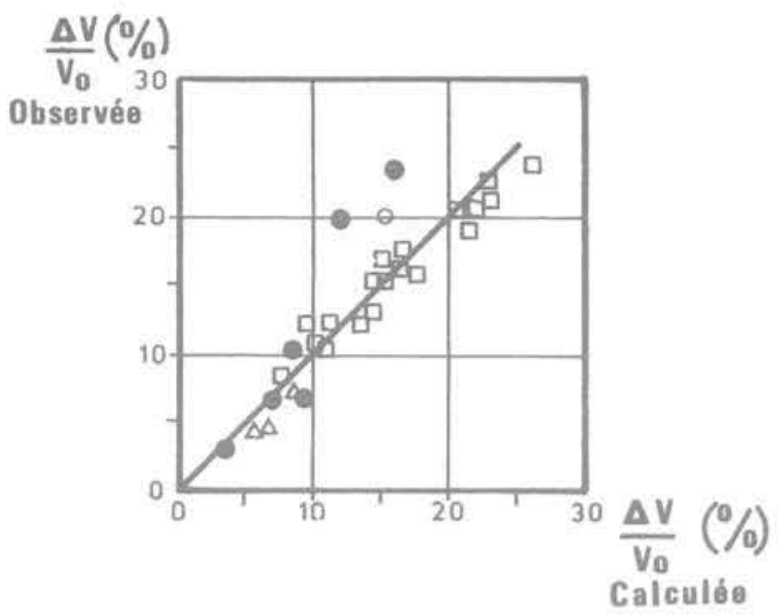

Fig. 9 Variations volumiques calculées et observées

Le résultat final du travail de $\mathrm{M}$. Alberro est un ensemble d'expressions reliant les variations volumiques des sols de gonflement ou de retrait à des paramètres simples de comportement tels que l'indice de plasticité, la teneur en eau initiale, le poids volumique initial, la limite de retrait, etc. Pour illustrer l'intérêt de ces expressions, la figure 9 montre les résultats obtenus pour la prévision des gonflements des sols utilisés dans la construction routière au Mexique et la comparaison avec les valeurs mesurées en laboratoire. Les expressions de M. Alberro ont été publiées dans les comptes rendus de la Conférence Panaméricaine de Mécanique des Sols et des Fondations qui a eu lieu récemment à Lima.

\section{Développement d'un modèle mathématique pour le phénomène de fissuration longitudi- nale}

A partir des recherches financées par le Secretaria de Asentamientos Humanos y Obras Publicas pour être effectuées par I'Instituto de Ingenierıa de I'Université Nationale, ces deux organismes ont mis au point et développé ensemble un modèle physicomathématique de la fissuration longitudinale des remblais.

Pour cela il était nécessaire d'étudier :

a) la distribution des contraintes et des déplacements induits dans un remblai lorsque l'on impose des déformations de dilatation ou de contraction au remblai ou au sol d'assise,

b) les déformations volumiques des sols en fonction de leur teneur en eau et de leur densité initiales.

c) la distribution et la variation de la teneur en eau des sols constituant le remblai et le terrain de fondation lorsque des variations saisonnières ont lieu.

Pour cette étude on a utilisé la méthode des éléments finis pour déterminer les déplacements, les contraintes effectives et les pressions interstitielles produites par les variations volumiques dans le sol. Le maillage était constitué par des triangles. La formulation matricielle du problème comprenait :

- une matrice de rigidité pour l'ensemble des éléments, en terme de contraintes effectives, 
- une relation matricielle entre les déformations volumiques des triangles et les déplacements des nœuds.

- une relation matricielle entre le volume de chaque triangle et la pression interstitielle,

- la matrice du poids propre et des surcharges.

- la matrice des déformations volumétriques imposées aux éléments.

Un programme de calcul pouvait alors être rédigé; en lui donnant les gonflements et les retraits des matériaux du remblai et du sol d'assise, on peut calculer la distribution des contraintes de traction engendrées.

Ce modèle a bien évidemment une double utilité, comme c'est souvent le cas dans ce genre de simulation des phénomènes physiques : d'une part il est possible de calculer les variations volumiques à partir de données réelles et avoir une idée des contraintes de traction et de leur distribution dans l'ouvrage; d'autre part, dans le cas d'un projet lorsqu'on connaît les propriétés des matériaux, il est possible de faire une estimation des variations volumétriques à partir des critères indiqués, et le modèle permet'de se faire une idée des contraintes de traction qui risquent de se produire et de leur distribution. Au-delà de ces applications évidentes, le modèle en a d'autres qui ne sont pas moins utiles : grâce aux facilités de calculs des ordinateurs et en donnant des valeurs arbitraires aux paramètres, on peut se rendre compte du poids de chacun des paramètres et leurs relations. Grâce à cela les critères du projet gagnent en finesse et en précision.

En termes généraux, les gonflements et retraits maximaux qui peuvent être atteints dans un problème réel sont de l'ordre de $20 \%$ et les valeurs minimales sont nulles. Curieusement pour des calculs réalisés avec des données réelles, le modèle conduit à des contraintes effectives maximales de traction qui vont jusqu'à 7,5 $\mathrm{MPa}$, ce qui explique largement les phénomènes de fissuration observés.

Les figures 10 à 12 représentent les contraintes de traction (en $\mathrm{MPa}$ ) données par le modèle, en supposant une zone de gonflement au centre et au-dessous du remblai et une zone de retrait dans les talus et dans le sol d'assise non protégé par le remblai lui-même. Sur chaque figure on peut voir l'amplitude des gonflements et des retraits.

D'après le modèle, il apparaît que :

1) le maximum du retrait a plus d'influence que le maximum du gonflement pour le développement de la fissuration,

2) les variations volumiques différentielles sont plus significatives dans le corps du remblai que dans le sol d'assise.

Ces deux conclusions ont été corroborées par les observations sur chantier. Les fissures apparues dans les remblais constitués de sols fins posés sur des roches, ce qui représente des cas tout à fait exceptionnels, sont toujours très fines car il n'y a pas de mouvement d'eau en provenance du sol d'assise, ce qui est à l'origine des plus fortes variations volumiques.

\section{Solutions proposées au problème de la fissuration}

II y a évidemment deux types de problèmes importants :

a) les remèdes à apporter à des remblais existants fissurés,

b) les projets de nouveaux remblais pour lesquels le phénomène de fissuration n'aura pas lieu ou pour que ces remblais soient protégés contre son développement.

Dans le premier cas on a retenu deux solutions. La première consiste à élargir la section du remblai de 2 à $3 \mathrm{~m}$ de chaque côté, ce qui n'empêche pas la formation de fissures, mais qui change leur position et les place en un lieu où elles ne risquent pas d'induire de plus grands dommages; une autre solution consiste à protéger le remblai en superposant des talus sur ceux qui existent, mais avec une plus grande pente (de $3: 1$ à $5: 1$ ). Cette deuxième solution s'est révélée plus économique et donne de meilleurs résultats que la première; la fissuration se produit alors sur les talus recouverts où le dommage produit est minimal, spécialement lorsqu'il y a de la végétation, ce qui élimine la nécessité de l'entretien. La végétation offre ainsi plusieurs avantages et peut même résoudre les petits problèmes.

Pour les nouveaux remblais une solution immédiate consiste à les protéger avec des matériaux non sensibles aux dilatations volumiques, cependant cette

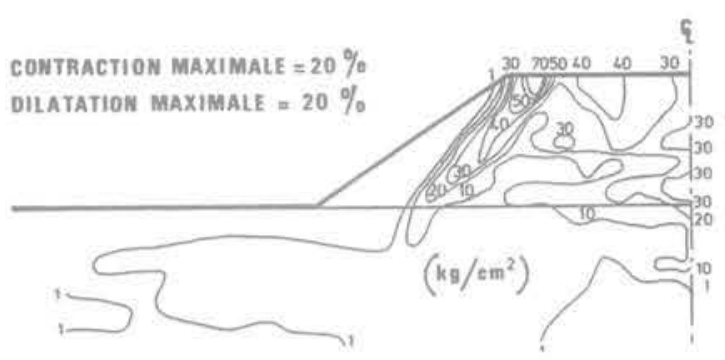

Fig. 10 Contraintes principales de traction

Fig. 11 Contraintes principales de traction
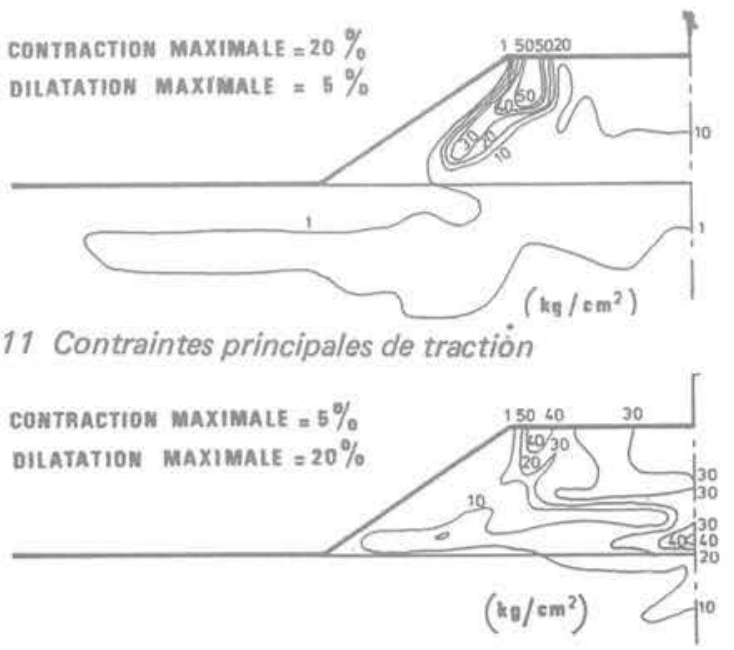

Fig. 12 Contraintes principales de traction 
solution n'est pas toujours en bon voisinage avec la nécessité de minorer le prix de la construction des routes à faible trafic. La mise en place d'une couche très poreuse de 20 à $25 \mathrm{~m}$ d'épaisseur pour * couper la capillarité" s'est toujours montrée comme une solution sûre et assez fiable, mais également coûteuse et malheureusement injustifiée pour les routes modestes. L'emploi de nappes en films plastiques se trouve actuellement dans sa première phase expérimentale au Mexique. Quelques nouveaux remblais ont été construits en observant ces règles de protection pour corriger la fissuration des remblais existants.
Un dernier commentaire en ce qui concerne la stabilisation des sols au moyen d'additions en faibles pourcentages de divers types de sols. Les résultats de cette excellente et économique méthode sont très bons et il est recommandé que le projeteur les garde en tête comme une solution éventuelle.

Les diverses autoroutes et sections expérimentales qui existent actuellement au Mexique permettront de comparer la valeur de ces différentes solutions au problème de la fissuration. 